\title{
HUMAN PILOT STUDY OF BARE-METAL STENT: INC-01
}

\author{
Arturo Abundes-Velasco ${ }^{1}$, Jorge Gaspar-Hernández ${ }^{2}$, Marco A. Martínez-Ríos $^{3}$, \\ Félix Damas-De los Santos ${ }^{3}$, Yigal Piña-Reyna ${ }^{3}$, Eduardo Arias-Sánchez ${ }^{3}$, José L. Romero-lbarra ${ }^{4}$,

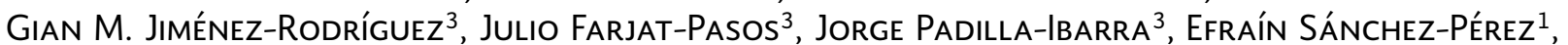 \\ Juan P. Sánchez-luna ${ }^{2}$, Ramsés Galaz-MÉndez ${ }^{5}$, Paola Ulacia-Flores ${ }^{6}$, Marco M. Arai-Ito $^{1}$, \\ David A. Rodríguez-Parra ${ }^{1}$, Sebastián Aceves-Díaz González ${ }^{1}$, and Marco A. Peña-Duque ${ }^{1}$
}

${ }^{1}$ Department of Innovation and Technology; ${ }^{2}$ General Directorate, and ${ }^{3}$ Department of Interventional Cardiology, Instituto Nacional de Cardiología Ignacio Chávez, Mexico City; ${ }^{4}$ Department of Cardiology, Hospital Star Médica, Querétaro, Qro.; ${ }^{5}$ Galaz Science and Engineering Biomedical, Sonora, Son.; ${ }^{6}$ Department of Biomedical Engineering, Instituto Tecnológico de Estudios Superiores de Monterrey, Mexico City, Mexico

\begin{abstract}
Background: The cost of performing a percutaneous coronary intervention is considerably high for the patient as well as for health systems, which have promoted the development of local technology to help meet the need for these devices. Methods: The INC-01 bare-metal stent was developed at the National Institute of Cardiology in Mexico City and was first implanted on porcine models with technical success in $100 \%$ of the evaluated parameters. Presentation of Cases: We present the first three cases of patients with ischemic heart disease, to whom the INC-01 bare-metal stent was implanted. Intracoronary ultrasonography was performed post-stent implantation, showing all the characteristics of implant success during evaluation and clinical follow-up. Conclusions: Angiography and intracoronary ultrasound were carried out demonstrating that the INC-01 bare-metal stent has physical, biological, and histological characteristics similar to those found in commercial metallic stents. (REV INVEST CLIN. 2020;72(1):32-6)
\end{abstract}

Key words: Bare-metal stent. Coronary angiography. Intracoronary ultrasound.

\section{INTRODUCTION}

The cost of performing a percutaneous coronary intervention is highly variable because a large number of materials and human resources are to be considered, the stent is a fundamental factor. In developing countries, the cost of this technology is expensive for the patient as well as for the health systems, which have promoted the development of local technology to help meet the requirements of these devices ${ }^{1}$. Recently, several countries have started developing their
*Corresponding author:

Marco A. Peña-Duque

E-mail: marcopduque@gmail.com
Received for publication: 16-07-2019

Approved for publication: 17-09-2019

DOI: $10.24875 / R I C .19003182$

0034-8376 / (c) 2019 Revista de Investigación Clínica. Published by Permanyer. This is an open access article under the CC BY-NC-ND license (http://creativecommons.org/licenses/by-nc-nd/4.0/). 
Figure 1. (A) Finite element tests by computer. (B) Thrombotic occlusion of the anterior descending coronary artery (red arrow). (C) Angiography after implanting a $3.5 \times 18 \mathrm{~mm}$ INC-01 bare-metal stent model (red arrow). (D) Intravascular ultrasound. In the image on the left, the distal reference diameter is observed and in the image on the right, the stent implanted with adequate expansion and apposition.
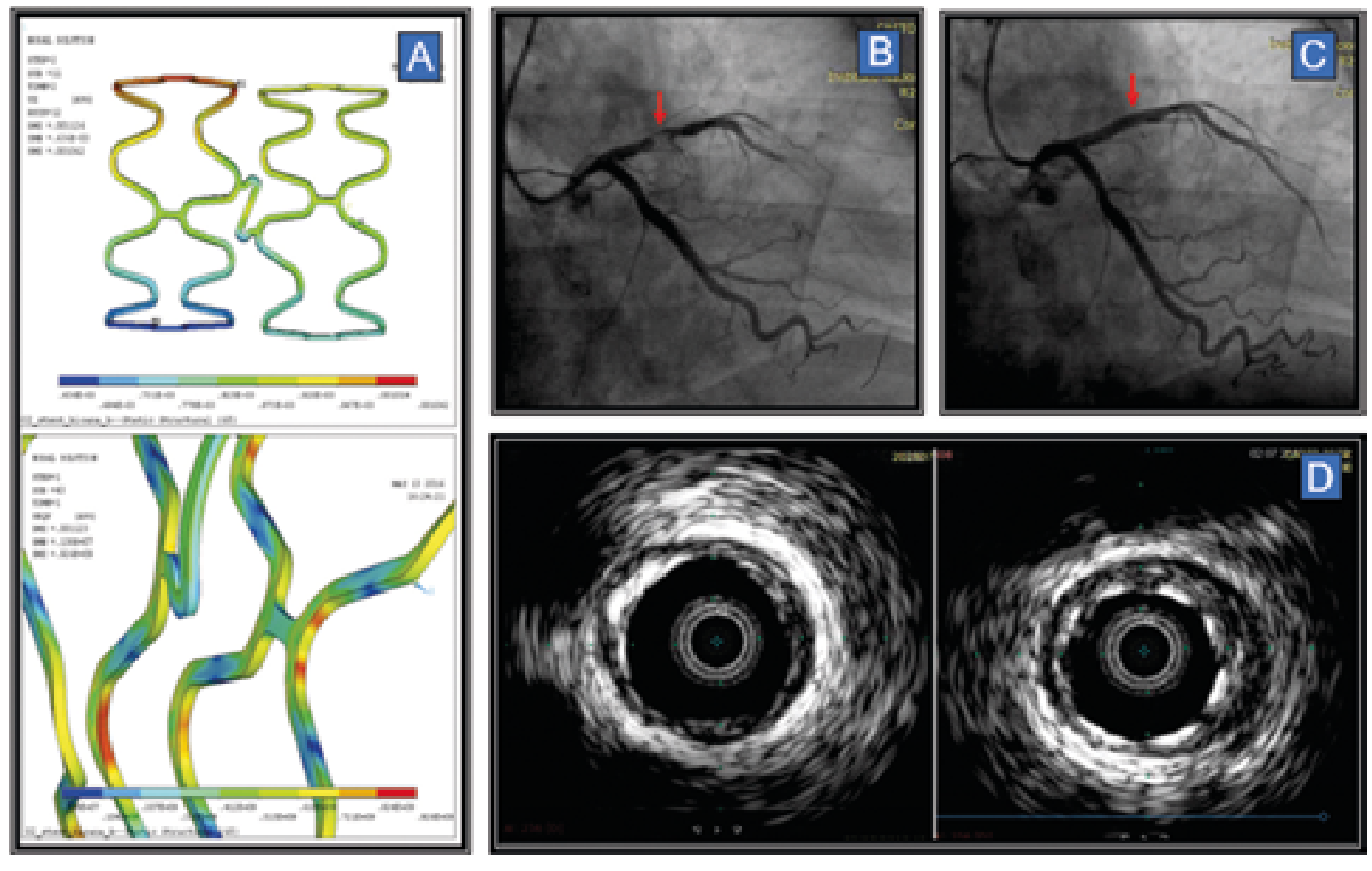

own stents due to the high cost of importing and distributing this technology $y^{2-4}$.

We have an extensive experience on successfully working with platinum/iridium stents manufactured by hand for coronary, aortic, and peripheral arteries ${ }^{5-7}$. As of 2014, a group from the National Institute of Cardiology (INC) associated with GSE Biomedical and the Technological Institute of Higher Studies of Monterrey (ITESM), all in Mexico, worked on the design and development of a Mexican coronary stent, using as a guide the methodology based on "quality function deployment." We compared the needs of the interventional cardiologist as the user, against the technical or engineering requirements, and compared with the maximum goal to be achieved considering the biophysical characteristics of commercial stents. In the design process, we agreed that among other characteristics, the INC-01 stent should have a cobalt platform and chrome L605 (Co-Cr), according to the international standard with struts of $80 \mu \mathrm{m}$ thickness, expanded by balloon, and with the performance equaling that of the second-generation stent or higher as per to the requirements of the ISO (ISO 25539-2: 2008) (Fig. S1). Design and testing proposals were made to select the geometric design of the stent based on finite element tests by computer (Fig. 1A).

\section{METHODS}

\section{In vitro tests}

About 15 in vitro tests were carried out according to the ISO standard (radial force, flexibility, elongation deformation, and longitudinal shortening, among others) and they showed adequate and consistent characteristics with the computational tests ${ }^{8}$. 
Figure 2. (A) On the left, lesion in the proximal segment of the anterior descending and the left main coronary artery (red arrow); on the right, post-implantation angiography is observed (red arrow). (B) The examination by intravascular ultrasound: on the left, adequate expansion of the stent is observed in the proximal segment of the anterior descending coronary artery; to the right, adequate stenting of the left main coronary artery. (C) On the left, a critical lesion was seen in the middle segment of the anterior descending coronary artery (red arrow); to the right, angiography after implantation of the INC-01 stent (red arrow). (D) On the left, the distal reference image is seen, and, on the right, the stent implanted properly.
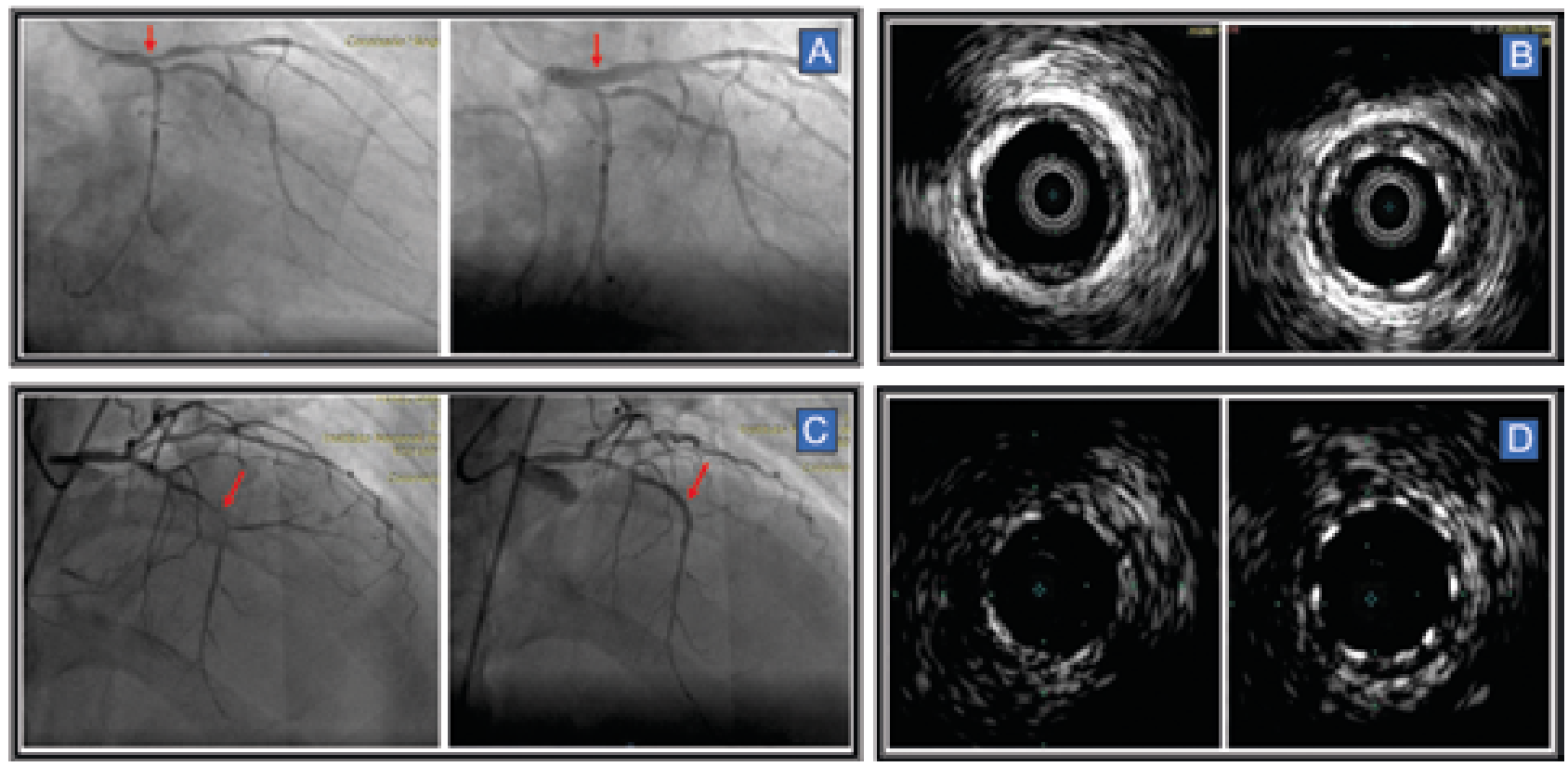

\section{In vivo tests}

Tests were performed on a porcine model with 10 live porcine specimens of the Yorkshire breed, obtaining technical success in $100 \%$ of the evaluated parameters. We demonstrated that the INC-01 bare-metal stent has physical, biological, and histological characteristics similar to those found in commercial metallic stents ${ }^{9}$.

With the above-mentioned results, and after submitting the human protocol for review to the Medical Ethics and Research Committee, the implementation of a first-in-man study was decided with prior informed consent from patients and following the recommendations of the Declaration of Helsinki.

\section{PRESENTATION OF CASES}

With prior informed consent, the INC-01 bare-metal stent was implanted in three patients from the INC following the international standards of care for ischemic heart disease. They were performed by radial access in two cases and femoral in one. Adequate stent expansion was confirmed by an intracoronary ultrasound with a $40 \mathrm{MHz}$ Volcano ${ }^{\circledR}$ probe. The patients were followed up every week for 1 month, then by telephone call every month, and finally by an inperson appointment after 6 months to perform coronary angiography and coronary ultrasound.

\section{Case 1}

A 67-year-old man, an active smoker but without chronic degenerative diseases, was admitted with a diagnosis of acute anteroseptal myocardial infarction. The coronary angiography identified thrombotic occlusion of the anterior descending artery in its proximal segment (Fig. $1 \mathrm{~B}$ and C). Pre-dilation with a $3.0 \times 20 \mathrm{~mm}$ conventional balloon was performed, and subsequently, a $3.5 \times 18 \mathrm{~mm}$ INC-01 bare-metal stent was implanted at nominal pressure, confirming adequate expansion and apposition of it by intracoronary ultrasound (Fig. 1D). The patient was discharged $72 \mathrm{~h}$ after coronary intervention. 


\section{Case 2}

A 64-year-old man, diabetic with hypercholesterolemia and an active smoker, was admitted with a diagnosis of inferior non-reperfused myocardial infarction. Coronary angiography was performed, finding trivascular coronary disease with involvement of the left main coronary artery, with failed angioplasty to the right coronary artery. The heart team considered the case unsuitable for revascularization surgery, so it was decided to perform percutaneous coronary revascularization. Intracoronary ultrasound was performed, and it was decided to implant a $3.5 \times 18 \mathrm{~mm}$ INC-01 bare-metal stent at nominal pressure to impact it at 14 atmospheres ( $4.11 \mathrm{~mm}$ ) (Fig. 2A); intravascular ultrasound (IVUS) was also performed, thereby confirming adequate expansion and complete apposition (Fig. 2B). Furthermore, another $2.5 \times 24$ $\mathrm{mm}$ commercial bare-metal stent was implanted in the middle segment of the anterior descending artery, without any complications. The patient was closely monitored for $72 \mathrm{~h}$ and then discharged. No ischemia was found in the right coronary artery.

\section{Case 3}

A 38-year-old hypertensive man, with a history of non-reperfused anteroseptal myocardial infarction and coronary lesions in the anterior descending and right coronary arteries, was admitted for angioplasty scheduled after a nuclear medicine study that reported an anteroseptal infarction with anterior and inferior ischemia. Therefore, a $3.5 \times 18 \mathrm{~mm} \mathrm{INC-01}$ bare-metal stent was implanted in the middle segment of the anterior descending artery (Fig. 2C) at nominal pressure, and symmetrical expansion and apposition were corroborated by intracoronary ultrasound (Fig. 2D); a 3.0 drug-eluting stent was subsequently implanted $33 \mathrm{~mm}$ in the middle segment of the right coronary artery. The patient was closely monitored and was discharged home symptomless after $24 \mathrm{~h}$.

\section{Clinical follow-up}

At 10 months of follow-up, the three patients have remained alive. One of them was admitted with thoracic pain of prolonged exertion (not typical angina) and has been followed with cardiac rehabilitation. At present, he is symptomless without angina or equivalent, with functional Class I of the New York Heart Association Classification. The other two patients remained symptomless and without clinical events.

\section{DISCUSSION}

In this pilot study of the INC-01 bare-metal stent, the adequate functionality of the device was confirmed, with a satisfactory advancement profile, good navigation, radial strength, and symmetrical expansion. The stent had no complications in the immediate postimplantation period or short-term follow-up, with a strict monitoring of the clinical status of patients with periodic visits, so it is considered that at present, the INC-01 bare-metal stent results are very promising. Therefore, a clinical protocol in humans that include a larger sample size and follow-up at different points with angiography and IVUS to evaluate safety and efficacy of the stent has been designed and approved by the ethics and scientific committees of our institute. The protocol has been admitted by the COFEPRIS (regulatory body for the use of medicines and medical devices in our country), which is in accordance to good international manufacturing and sterilization practices.

This study is our first experience in human implantation of the INC-01 stent, where we only evaluated clinical data. However, it must be replicated in a larger sample, and necessarily, we should have a follow-up with angiography and IVUS.

\section{Supplementary data}

Supplementary data are available at Revista de Investigación Clínica online (www.clinicalandtranslationalinvestigation.com). These data are provided by the corresponding author and published online for the benefit of the reader. The contents of supplementary data are the sole responsibility of the authors. 


\section{REFERENCES}

1. Wadhera P, Alexander T, Nallamothu BK. India and the coronary stent market: getting the price right. Circulation. 2017;135:1879-81.

2. Qian J, Xu B, Lansky AJ, Yang YJ, Qiao SB, Wu YJ, et al. First report of a novel abluminal groove filled biodegradable polymer rapamycin-eluting stent in de novo coronary artery disease: results of the first in man FIREHAWK trial. Chin Med J. (Engl) 2012;125:970-6.

3. Xu B, Gao RL, Zhang RY, Wang HC, Li ZQ, Yang YJ, et al. Efficacy and safety of FIREHAWK ${ }^{\circledR}$ abluminal groove filled biodegradable polymer sirolimus-eluting stents for the treatment of long coronary lesions: nine-month angiographic and one-year clinical results from TARGET I trial long cohort. Chin Med J (Engl). 2013;126:1026-32.

4. Asano T, Suwannasom P, Katagiri Y, Miyazaki Y, Sotomi Y, Kraak $\mathrm{RP}$, et al. First-in-man trial of SiO2 inert-coated bare metal stent system in native coronary stenosis-the AXETIS FIM trial. Circ J. 2018;82:477-85
5. Abundes-Velasco A, Rivera-Arellano J, Arizmendi-Uribe E, Ledesma-Velasco M, Farell-Campa J, Montoya-Guerrero S. Resultados inmediatos y a largo plazo del implante de un nuevo stent coronario de platino (stent Atlas) en pacientes con enfermedad arterial coronaria. Rev Esp Cardiol. 2002;55:1205-8.

6. Abundes-Velasco A, Zabal-Cerdeira C, García-Montes JA, De los Ríos-lbarra MO, Gallegos JE, Peña GQ, et al. Treatment of seven patients with coarctation of the aorta treated using a mexicanmade platinum/iridium stent. Arch Med Res. 2007;38:853-7.

7. Abundes-Velasco A, Arizmendi-Uribe E, De los Ríos-lbarra MO, Estrada-Gallegos J, Peña-Quintana G. Results of the first 5 patients with iliac artery lesions treated by placement of a platinum/iridium stent of Mexican manufacture and literature review. Rev Mex Cardiol. 2010;21:49-56.

8. Abundes-Velasco A, Romero-Ibarra JL, Sandoval-Jones JP, GalazMéndez R, Ulacia-Flores P, Peña Duque MA, et al. Diseño y fabricación de un stent coronario e inicio de pruebas en animales de experimentación. Rev Mex Cardiol. 2016;27::(suppl. 5):226-27.

9. Abundes-Velasco $A$, Rodríguez-Barriga $E$, Custodio-Sánchez $P$, Romero-lbarra JL, Galaz-Méndez R, Ulacia-Flores P, et al. Experiencia fase animal del stent coronario INC. Arch Cardiol Mex. 2017;87:79. 